\title{
ESTUDO DE CASO SOBRE O GERENCIAMENTO DOS SERVIÇOS DE TI EM UM ÓRGÃO PÚBLICO
}

\author{
CASE STUDY ON THE MANAGEMENT OF IT SERVICES IN A PUBLIC \\ BODY
}

Franciély Aparecida Vieira Silva, Helton Molina Sapia, Haroldo Cesar Alessi, Rogerio Marcus Alessi, Glauco Antonio Ruiz, João Cezario Giglio Marques, Dione Jonathan Ferrari

Universidade do Oeste Paulista - UNOESTE, Especialização em MBA em TI, Presidente Prudente, SP.

E-mail: franciely1128@hotmail.com

RESUMO - A ITIL é o acrônimo de "Information Technology Infrastructure Library" ou Biblioteca de Infraestrutura de Tecnologia da Informação. Objetiva proporcionar maior satisfação dos clientes e usuários e aumentar a eficiência e a eficácia dos serviços prestados, o que resulta na diminuição de custos e no aumento da qualidade dos serviços de TI. Este trabalho apresenta um estudo de caso sobre o gerenciamento de serviços de TI em um órgão público. Por meio de análises obteve-se o conhecimento de como eram realizadas as rotinas do departamento de TI na organização. Foram coletadas as informações por meio de visitas nos departamentos da prefeitura, podendo observar muitos pontos que deveriam ser mudados e melhorados, justificando assim a necessidade do desenvolvimento deste trabalho. Foi apresentada uma visão geral dos resultados obtidos pelo desenvolvimento do estudo de caso, mostrando as mudanças que podem ser realizadas para melhorar os serviços prestados pelo departamento de $\mathrm{TI}$, como também melhorar os serviços dos demais setores da organização, sugerindo soluções para os principais pontos problemáticos, de forma que as mesmas sejam implantadas de maneira gradual para se obter um melhor resultado. Por fim, o trabalho será apresentado para o chefe de gabinete da prefeitura para que seja aprovado e colocado em prática.

Palavras-chave: ITIL; Gerenciamento de Serviços de TI.

ABSTRACT - ITIL is the acronym for the Information Technology Infrastructure Library, aimed at providing greater customer and user satisfaction and increasing the efficiency and effectiveness of the services provided, resulting in reduced costs and increased Quality of IT services. This paper presents a case study on the management of IT services in a public agency. We analyzed and had knowledge of how the routines of the IT department in the organization were performed. The information was collected through visits to the departments of the city hall, and many points could be observed that should be changed and improved, thus justifying the need to develop this project. This paper presents an overview of the results obtained by the 
development of the case study, showing the changes that can be made to improve the services rendered by the IT department, as well as improving the services of the other sectors of the organization, suggesting solutions for the main Problem points so that they are gradually implemented to achieve a better result. Finally, the work is presented to the company's management to be approved and put into practice.

Keywords: ITIL; IT Service Management. 


\section{INTRODUÇÃO}

ITIL é o acrônimo de "Information Technology Infrastructure Library" ou Biblioteca de Infraestrutura de Tecnologia da Informação. Visa proporcionar maior satisfação dos clientes e usuários e aumentar a eficiência e a eficácia dos serviços prestados, o que resulta na diminuição de custos e no aumento da qualidade dos serviços de TI (FERNANDES, 2014).

De acordo com Magalhães (2007), a ITIL sugere que as atividades de gerenciamento de serviços sejam estruturadas com base no ciclo de vida do serviço. Esse ciclo considera a 'vida' do serviço desde a sua concepção até a sua descontinuação.

Cada um dos cinco principais livros da biblioteca se refere a um estágio específico do ciclo de vida do serviço:

Estratégia de Serviço: esse é o estágio onde é definida a direção estratégica dos serviços de $\mathrm{TI}$, quem são os seus clientes e quais serviços serão disponibilizados para eles.

Desenho de Serviço: desenhar um novo serviço ou modificar um já existente visando sua entrada no ambiente produtivo.

Transição de Serviço: o estágio de transição de serviço ajuda a organização a planejar e gerenciar mudanças em serviços e implementar liberações no ambiente produtivo.

Operação de Serviço: nesse estágio são coordenadas e executadas as atividades e processos necessários para entregar os serviços aos clientes e usuários do negócio, e gerenciando os serviços nos níveis acordados.

Melhoria continuada de serviço: o propósito da melhoria continuada de serviço é alinhar e realinhar continuamente os serviços de TI de acordo com as necessidades do cliente, identificando e implementando melhorias aos serviços de TI que suportam os processos de negócio.

De acordo com o ITSMF (2017), dentre os principais benefícios do uso do modelo ITIL V3 podemos mencionar: Alinhamento de $\mathrm{TI}$, seus serviços e riscos com as necessidades do negócio; Níveis de Serviço (SLA) negociáveis; Processos consistentes e previsíveis; Eficiência na entrega de serviço; Serviços e Processos mensuráveis e passíveis de melhorias; Otimização da experiência do cliente e uma linguagem comum.

O objetivo principal deste estudo, foi apresentar um estudo de caso sobre o Gerenciamento de Serviços de TI no setor da Tecnologia da Informação de um órgão público, na Prefeitura Municipal de Santo Inácio, aplicando os processos e funções da ITIL no setor. Onde esse estudo será realizado com o auxílio das normas e série, 
para assim tornar uma organização melhor, através das melhorias implementadas com o auxílio da ITIL.

\section{METODOLOGIA}

O estudo foi realizado na Prefeitura Municipal, além de destacar a importância da Tecnologia da Informação para a execução dos processos chaves observou-se muitas falhas indicando a necessidade de melhorias. Atualmente os serviços prestados pela Tecnologia da Informação são feitos conforme a demanda e a necessidade de cada setor.

Os serviços prestados, relacionados a TI, são os seguintes: (i) Infraestrutura e administração de rede (terceirizado); (ii) Consertos de hardwares; (iii) Instalações e atualizações de software; (iv) Desenvolvimento do site da prefeitura; (terceirizado); (v) Softwares terceirizados; (vi) Manutenção do site; (vii) Funcionamento da internet; (viii) Funcionamento dos computadores em todos os setores; (ix) Funcionamento de todas as impressoras em todos os setores.

Utilizando-se dos princípios da ITIL foi conduzida uma análise pontuando os serviços que estão em consonância com a estratégia de serviço, os serviços escolhidos foram definidos baseando-se no desenho de serviço, para os serviços que ainda não estão em operação foi apresentada a transição de serviço e para os demais foi apresentada a operação do serviço, por fim pretende-se criar um ambiente favorável a melhoria continuada de serviço uma vez que tanto a organização como a tecnologia da informação estão constantemente em evolução.

Durante a condução destes trabalhos foram identificados alguns problemas, são eles: (i) Não existe um software para gerenciar os incidentes ocorridos nos setores. De forma que para entrar em contato com a T.I. é preciso ligar no celular do funcionário e ele quem decide quem atender primeiro; (ii) Não é gerado uma ordem de serviço; (iii) Todos os funcionários de todos os setores, desde que possuam algum computador, podem instalar e desinstalar qualquer software que desejar; (iv) Alguns computadores não possuem antivírus; (v) Todos os sites são liberados em todos os computadores; (vi) A maioria das máquinas não são catalogadas, ou seja, não se sabe qual máquina e qual configuração pertence a um determinado setor; (vii) Demora muito tempo para ser dado assistência, e devido não ter uma prioridade, na maioria das vezes o departamento que precisaria ser atendido primeiro, acaba sendo atendido por último; (viii) Falta de comprometimento da "equipe" da T.I., muita demora principalmente no suporte; (ix) Quando alguma peça de uma determinada máquina quebra, ao invés de 
solicitar outra peça para realizar o conserto, é retirada a peça da máquina que estava funcionando corretamente e realiza a troca, ficando então, essa máquina parada, sem utilização. (x) Não se tem o controle das peças, não se sabe se um determinado item de configuração foi comprado, ou se está em uso, ou fora de uso, ou em reparo, ou até sem solução de conserto. (xi) Falta a implantação de um sistema de abertura de chamados, para tornar os serviços de suportes mais rápido e eficiente. (xii) $\mathrm{O}$ departamento de TI não possui um espaço físico, ou seja, não tem uma sala climatizada e com acesso controlado para instalação do rack que abrigar os equipamentos.

\section{RESULTADOS}

De acordo com as orientações da ITIL e das informações obtidas através do estudo sobre o gerenciamento dos serviços de T.I. dentro da prefeitura, foi possível aplicar vários processos da ITIL indicando ações para que os problemas mencionados possam ser solucionados.

Implantar o Data Center: local físico, onde terá todos os equipamentos de $\mathrm{TI}$ da prefeitura instalados, inclusive o local de trabalho do responsável pela T.I.

Gerenciar os Incidentes e demais demandas de usuários (servisse desk): Implantar um software de Gerenciamento de Incidentes e gestão dos problemas de inventário de T.I., sugere-se o software chamado GLPI, pois ele ajudará ainda em outros pontos a serem desenvolvidos.

Implantar o Gerenciamento da Configuração: ele é responsável por um Item de Configuração (IC), que permite o encaminhamento correto do incidente, pois detalha as informações que descrevem o hardware e software da prefeitura, identificando se existe alguma coisa errada em relação aos itens de configuração (switch, link internet, impressora, mouse, teclado, etc), usadas nos serviços.

Implantar o Gerenciamento de Evento: com a implantação deste gerenciamento, a TI passará a ter o controle dos softwares instalados nas máquinas, quais são licenciados, e quais estão sem a licença.

Cumprimento de Requisição: a maioria das requisições refere-se a pequenas mudanças que um usuário desejar realizar, ou seja, de baixo risco e baixo custo, o mesmo deverá abrir um chamado solicitando essa requisição, (conforme modelo de requisição pré-definido) e a central de serviços irá fazer o monitoramento, escalonamento e despachar os processos de gerenciamento de mudança e gerenciamento de incidente.

\section{Implantar o Gerenciamento de} Mudança: De acordo com levantamento feito nos departamentos da prefeitura, são solicitadas mudanças (atualização de 
software, upgrade em computador, etc) em algum serviço de $\mathrm{TI}$, mas na maioria das vezes essas solicitações não são atendidas, e quando alguma é realizada, não é feito uma Requisição de Mudança (RDM). Então, a partir da aplicação desse processo da ITIL para cada mudança solicitada será gerada uma RDM, que registra todos os detalhes da mudança em qualquer item de configuração (IC), tornando-se um padrão para resolver determinado problema, podendo ser observado o seu impacto antes de realizar a mudança, assim é feita a análise da mudança e se impactar de forma positiva a mudança é testada e implementada trazendo como benefício a melhoria nos serviços. Caso seja detectado que irá impactar de forma negativa, a mudança não é implantada.

A partir disso, toda vez que uma determinada mudança for solicitada, é verificada se já foi implantada anteriormente, e então utiliza-se esse documento como um modelo padrão para realizar determinada mudança, não se esquecendo da definição da prioridade da mudança: imediata, alta, média e baixa.

\section{Gerenciamento de Configuração e}

Ativo de Serviço: aplicando esse processo da ITIL será possível fornecer informações completas e atualizadas das configurações dos ativos de $\mathrm{TI}$, como: hardware, software e documentos afins.
Implementação do Gerenciamento da Segurança da Informação: os setores da prefeitura em geral, estão necessitados de aplicações de políticas de segurança. Como foi observado, vários problemas relacionados à segurança da informação foram encontrados, como por exemplo, muitas máquinas não possuem antivírus instalado ou ainda os computadores de todos os setores, sem exceção, possuem livre acesso a todos os sites da internet.

\section{Prioridade de Aplicação dos Processos da ITIL}

Após realizar o levantamento dos problemas que existem relacionados ao uso da Tecnologia da Informação na Prefeitura e quais processos devem ser aplicados para corrigi-los, serão mencionados logo abaixo a prioridade de implantação:

A sequência de implantação dos serviços seria da seguinte forma:

Primeiramente implantar o Data Center, para a prefeitura passar a ter um ambiente projetado para concentrar servidores, equipamentos de processamento e armazenamento de dados, e sistemas de ativos de rede, como switches, roteadores e outros.

A seguinte implantação seria de um Service Desk, para assim fazer o gerenciamento dos incidentes de forma correta, passando a ser um meio único para 
gerenciar as requisições e chamadas de serviços.

Implantar o Gerenciamento de Mudança, passando a controlar todas as mudanças solicitadas, sendo analisadas e implantadas para resultar em melhorias a prefeitura.

Implementar o Gerenciamento da Segurança da Informação, passando a aplicar políticas de segurança dentro da organização.

Realizar a implantação do Gerenciamento de Ativo de Serviço para ter controle sobre o hardware e o software.

Após a implementação do processo citado acima: "Gerenciamento de Ativo de Serviço", deve-se aplicar o Gerenciamento da Configuração, para assim a organização ter detalhadamente os hardwares e os softwares usados nas máquinas da prefeitura.

Aplicar o processo de Gerenciamento de Evento, controlando as licenças de softwares das máquinas em geral.

E por último, fica para ser implantado o Cumprimento da Requisição.

\section{DISCUSSÃO}

Conforme exposto na introdução, segundo Magalhães (2007), a tecnologia da informação é hoje fator-chave de sucesso para qualquer organização. Muitos especialistas veem o departamento de T.I. como "uma empresa dentro de uma empresa", atuando como prestadora de serviços para as demais áreas da organização.

Diante desse panorama, de acordo com Teruel (2014), o Gerenciamento de Serviços de T.I. tem papel fundamental para que as organizações alcancem suas metas e objetivos de negócio, através de processos e métodos bem definidos, que tenham eficiência comprovada no que tange à Gestão de infraestrutura e Serviços de TI. Um exemplo disso é a ITIL, base para as considerações referentes ao tema de Gerenciamento de Serviços de TI utilizadas neste estudo.

Pode-se afirmar que o Gerenciamento de Serviços de $\mathrm{TI}$ aqui proposto para a prefeitura tem por objetivo prover e garantir a qualidade, através de atividades e processos destinados aos usuários dos vários setores da prefeitura.

Ao analisar os processos contidos no framework (ITIL), pode-se perceber que os conceitos de negócio, valor gerado ao negócio e satisfação do cliente estão muito evidentes e neste estudo buscou-se explorar esses conceitos por todas as etapas do Ciclo de Vida do Serviço sempre considerando a realidade do ambiente organizacional da prefeitura.

\section{CONCLUSÃO}

O foco deste estudo baseou-se na importância que Gerenciamento de Serviços 
de $\mathrm{Tl}$ tem sobre os serviços de uma organização, no caso, da prefeitura municipal de Santo Inácio. Com o objetivo de explanar como se dá o processo de geração de valor aos serviços de Tecnologia da Informação, foi apresentado o framework ITIL, o conjunto de melhores práticas mais consagrado no mercado para o Gerenciamento de Serviços de TI.

Através de seus processos, que agrupados representam o Ciclo de Vida de um Serviço, foram discorridas todas as etapas no processo de geração de valor para um serviço conforme os livros ITIL. De acordo com Oliveira (2013), o objetivo da ITIL é fornecer as melhores práticas para a Gestão de Serviços de TI de uma maneira que possam ser aplicadas de diversas formas, adaptando-se a necessidade de cada negócio. Com isso, busca-se trazer a melhoria de satisfação, redução de custos, melhor comunicação, padrões bem estruturados para processos, aumento de produtividade e qualidade no serviço final, contribuindo para que a organização obtenha bons resultados.

Conclui-se com este trabalho, que ao implementar esses processos da ITIL, mencionados no RESULTADOS, os problemas nos serviços de Tecnologia da Informação da Prefeitura Municipal de Santo Inácio serão tratados, trazendo benefícios para a organização, e principalmente, para os setores que utilizam diretamente esses serviços, mas também para departamento de T.I., que a partir disso, conseguirá realizar o tratamento dos incidentes/problemas de uma forma eficaz, além de tornar-se um departamento organizado..

\section{REFERÊNCIAS}

FERNANDES, AGUINALDO ARAGON; ABREU, VLADIMIR FERRAZ DE. Implantando a Governança de $\mathrm{TI}$ - da estratégia a gestão de processos e serviços. 4a ed. Rio de Janeiro: Brasport, 2014.

ITSMF. itSMF International - The IT Service Management Forum. ITIL. Disponível em < http://www.itsmfi.org/forums/Posts.aspx?to pic $=1131678>$. Acesso em: 27 jul. 2017.

MAGALHÃES, IVAN LUIZIO; BRITO, MALFRIDO. Gerenciamento de serviços de TI na prática - uma abordagem com base na ITIL, inclui ISO/IEC 20.000 e IT Flex. São Paulo: Novatec Editora, 2007.

OLIVEIRA, BRUNO SOUZA DE. Métodos ágeis e gestão de serviços de TI. Rio de Janeiro: Basport, 2013.

TERUEL, EVANDRO CARLOS. ITIL V3 Atualizada em 2011: conceitos e simulados para certificação da ITIL Foundation. Editora Ciência Moderna, 2014. 